\title{
Spatial Mapping of Electrostatics and Dynamics in Quantum Materials
}

\author{
Akshay Murthy, Stephanie Ribet, Roberto dos Reis and Vinayak Dravid \\ Department of Materials Science and Engineering, Northwestern University, United States
}

Two-dimensional (2D) material heterostructures have proven useful for a wide range of applications such as quantum and optoelectronic devices.[1,2] In these systems the charge transport characteristics across various interfaces play a critical role in determining device performance, yet the relationship between atomistic dynamics and macroscopic properties is commonly unclear. This makes it difficult to identify the ideal methods for improving performance and reliability. To this end, in situ electrical biasing scanning/transmission electron microscopy (S/TEM) is a particularly promising tool for establishing structure-property relationships at the relevant length scales in such devices.[3] Furthermore, the versatile nature of S/TEM provides the possibility for detecting emergent phenomena in addition to structural information.[4] For instance, techniques such as conventional differential phase contrast [5] and first moment S/TEM [6] allow for relating the angular deflection of the electron probe to the gradient of the electrostatic potential in the sample plane.

Although, previous in situ demonstrations have been largely limited to individual 2D materials, we have recently developed a methodology for deterministic construction of $2 \mathrm{D}$ heterostructure devices for in situ electrical biasing S/TEM analysis.[7] By combining this method with first moment S/TEM, we produce a new platform technique that allows researchers to probe local electrical fields during electrical operation with atomic-scale precision. Using this method, it is possible to compare a priori electric field expectations with experimentally derived values to address questions regarding charge transport in nanoscale devices.

We use this platform to investigate a $\mathrm{MoS}_{2} /$ hexagonal $\mathrm{BN}(\mathrm{hBN})$ heterostructure. A scanning diffraction dataset taken from this sample is provided in Figure 1. Applying an external electric field leads to an appreciable angular deflection in the center of mass of the electron probe. The spatial variation in the calculated electric field and charge density maps (Figure 2) suggests that in the case of this heterostructure, charge carriers are injected through point sources such as local or atomic defects. The presence of this charge transport mechanism has implications for future electrical contact and Josephson junction applications. This work suggests that the use of phase contrast microscopy combined with an in situ analysis platform offers a route to identifying sources of performance degradation in a wide variety of analogous quantum and optoelectronic devices.

This material is based upon work supported by the National Science Foundation under Grant No. DMR1929356. This work made use of the EPIC, Keck-II, and SPID facilities of Northwestern University's NUANCE Center, which has received support from the SHyNE Resource (NSF ECCS-2025633), the IIN, and Northwestern's MRSEC program (NSF DMR-1720139). A.A.M. gratefully acknowledges support from the Ryan Fellowship and the IIN at Northwestern University. Research reported in this publication was supported in part by instrumentation provided by the Office of The Director, National Institutes of Health of the National Institutes of Health under Award Number S10OD026871. The content is solely the responsibility of the authors and does not necessarily represent the official views of the National Institutes of Health. This research was supported in part through the computational resources and staff contributions provided for the Quest high performance computing facility at Northwestern University which is jointly supported by the Office of the Provost, the Office for Research, and Northwestern University Information Technology. 


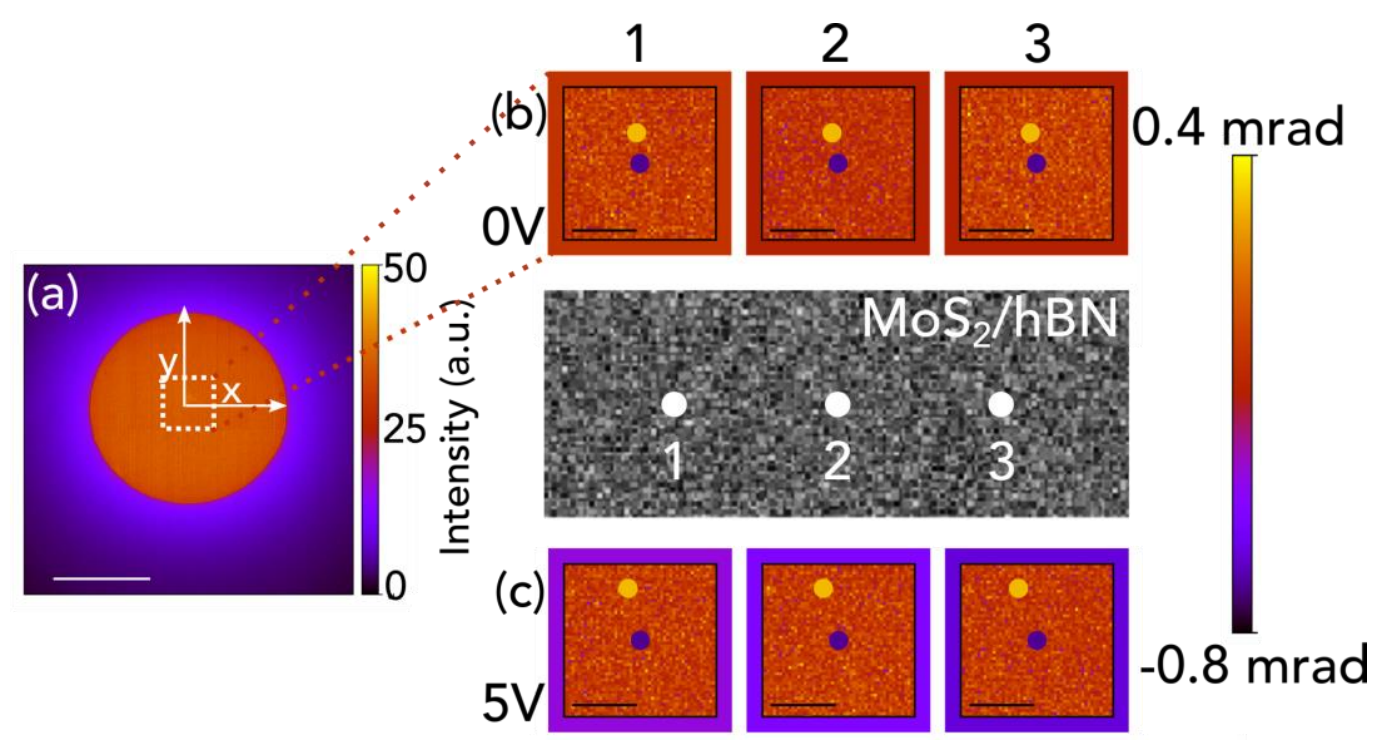

Figure 1. Scanning diffraction data taken from an $\mathrm{MoS} 2 / \mathrm{hBN}$ heterostructure. (a) convergent beam electron diffraction (CBED) pattern captured at point 1 marked on dark field TEM image. Colorbar intensity is provided in units of relative signal intensity. Scale bar in a represents $15 \mathrm{mrad}$. CBED patterns taken at positions 1,2, and 3 when an external bias of $0 \mathrm{~V}$ and $5 \mathrm{~V}$ is applied are provided in (b) and (c), respectively. The purple dots refer to the center of mass of the CBED pattern taken from vacuum, while the yellow dots represent the center of mass of the CBED pattern taken from each marked position on the sample. The border color represents the degree of probe deflection in the $\mathrm{x}$-direction. A clear shift in center of mass is observed as a function of electrical bias. Scale bars in b and c represent 2 mrad.
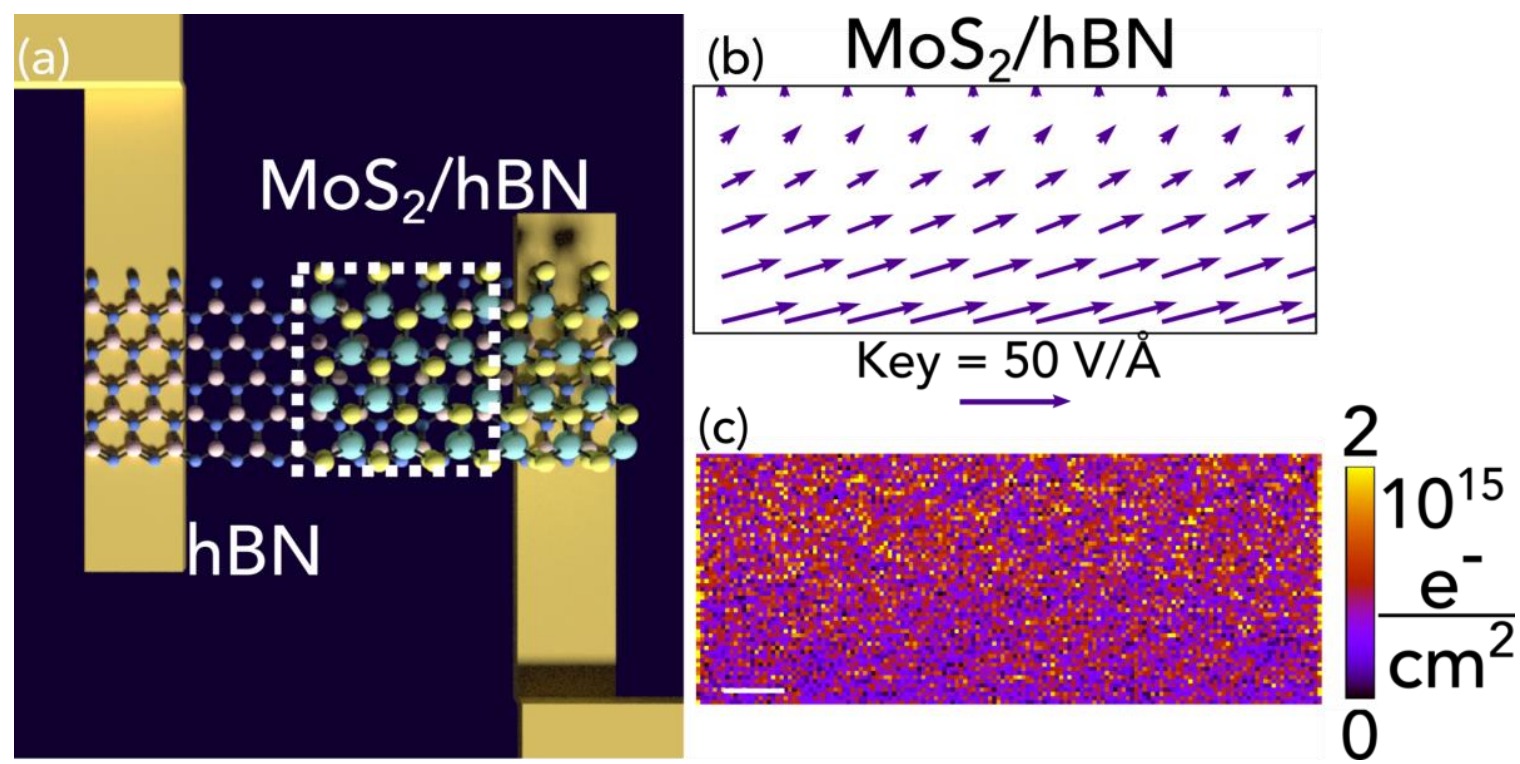

Figure 2. a) Plan-view schematic of the interface being examined. MoS $2 / \mathrm{hBN}$ region is highlighted. (b) electric field distribution and (c) charge density plot taken from the MoS2/hBN region when an external voltage of $5 \mathrm{~V}$ is applied. Scale bar represents $10 \mathrm{~nm}$.

References

[1] V E Calado et al, Nature Nanotechnology, 10 (2015), p. 761-764 
[2] F Wang et al, Nano Letters, 15 (2015). p. 7558-7566

[3] A A Murthy et al, ACS Nano, 14 (2020), p. 1569-1576

[4] S M Ribet, A A Murthy et al, Materials Today (2021) doi.org/10.1016/j.mattod.2021.05.006

[5] N Shibata et al, Nature Physics, 8 (2012). p. 611-615

[6] K Müller et al, Nature Communications, 5 (2014). p. 5653

[7] A A Murthy et al, arXiv, (2020), 2012.13842 